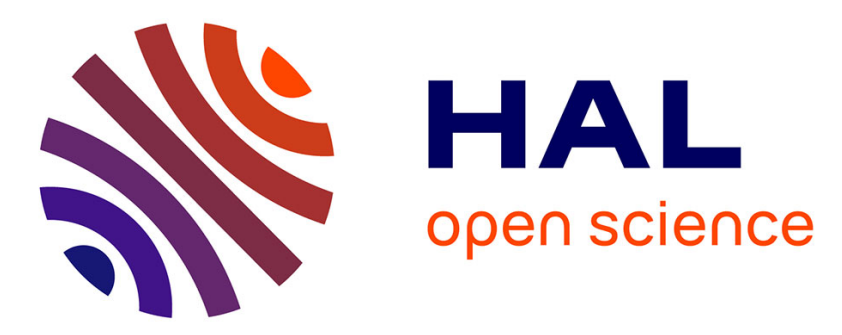

\title{
From strange to charmed baryons using two-flavour QCD
}

\author{
J. Carbonell, V. Drach, M. Papinutto
}

\section{To cite this version:}

J. Carbonell, V. Drach, M. Papinutto. From strange to charmed baryons using twoflavour QCD. 15th International QCD Conference, Jun 2010, Montpellier, France. pp.367-370, 10.1016/j.nuclphysbps.2010.10.095 . in2p3-00531322

HAL Id: in2p3-00531322

https://hal.in2p3.fr/in2p3-00531322

Submitted on 3 Nov 2010

HAL is a multi-disciplinary open access archive for the deposit and dissemination of scientific research documents, whether they are published or not. The documents may come from teaching and research institutions in France or abroad, or from public or private research centers.
L'archive ouverte pluridisciplinaire HAL, est destinée au dépôt et à la diffusion de documents scientifiques de niveau recherche, publiés ou non, émanant des établissements d'enseignement et de recherche français ou étrangers, des laboratoires publics ou privés. 


\title{
From strange to charmed baryons using two-flavour QCD
}

\author{
Jaume Carbonell $^{\mathrm{a}}$, Vincent Drach $^{\mathrm{b}}$, Mauro Papinutto $^{\mathrm{a}, * *},{\text { [ETM Collaboration }]^{* *}}$ \\ ${ }^{a}$ Laboratoire de Physique Subatomique et de Cosmologie, UJF/CNRS-IN2P3/INPG, 53 rue des Martyrs, F-38026 Grenoble, France \\ ${ }^{b}$ NIC, DESY, Platanenallee 6, D-15738 Zeuthen, Germany
}

\begin{abstract}
We compute the mass spectrum for strange and charmed baryons in the partially quenched approach using $N_{f}=2$ twisted mass QCD configurations. We investigate two main issues: the size of lattice artefacts using three values of the lattice spacing (the smallest of which is approximately $0.05 \mathrm{fm}$ ) and the dependence of baryon masses on meson masses. We thus perform a global fit in order to extrapolate simultaneously to the continuum limit and to the physical point. We obtain predictions for the $\Omega_{s s s}, \Xi_{d s s}, \Lambda_{u d s}, \Omega_{c c c}, \Xi_{d c c}, \Lambda_{u d c}$ masses.
\end{abstract}

Keywords: Lattice QCD, twisted mass, baryon physics

\section{Introduction}

Lattice regularization of QCD offers the unique opportunity of computing non-perturbative quantities like the hadron spectrum, decay constants, weak matrix elements and form factors from first principles. Using this regularization, the functional integral can be in fact evaluated numerically through Monte Carlo simulations. Numerical simulation are performed for a range of values of the lattice spacing $a$ and of the quark masses $m_{q}$ and then have to be extrapolated to the continuum limit and to the physical value of the quark masses.

The inclusion of (light) sea quarks in numerical simulations proves to be particularly challenging and in the past sea quark effects were often neglected, leading to the so-called quenched approximation. Nowadays simulations with two light degenerate sea quarks $\left(N_{f}=2\right)$ and in some cases including also the strange sea quark $\left(N_{f}=2+1\right)$ or both the strange and the charm sea quarks $\left(N_{f}=2+1+1\right)$ have become standard even though the light quarks are not yet as light as the physical up/down quarks. The difficulty in reaching the physical point is due to the slowing down of Monte Carlo simulations in the critical region and also to the fact that light quarks at the physical point require big volumes in order the observables not to be affected by large finite size effects.

\footnotetext{
*Speaker

** Preprint numbers: DESY 10-169, LPSC10155

Email addresses: jaume.carbonell@lpsc.in2p3.fr (Jaume Carbonell), vincent.drach@desy.de (Vincent Drach), mauro.papinutto@lpsc.in2p3.fr (Mauro Papinutto)
}

The inclusion of the strange sea quark, due to his heavier mass, is slightly less important and at present there are no striking examples where $N_{f}=2+1 / N_{f}=2+1+1$ simulations gives substantially different results with respect to the $N_{f}=2$ case. This is believed to be even more the case for the charm sea quark.

Valence quarks can be treated independently from the sea quarks and can be simulated with the same masses of the sea quarks or with different ones. One can even consider valence quarks which are not present in the sea, for example the strange and the charm quark in the case of $N_{f}=2$ simulation. This setup is called partially quenched approximation and we will use it in the present study.

Concerning heavy quarks (e.g. the bottom quark) the compton wavelength of the associated heavy-light meson is too small compared to present attainable lattice spacings. This means that heavy quarks feel discretization effects and related observables can be affected by large lattice artefacts. The charm quark mass is at the upper boundary of the range of masses that can be simulated at present for the coarsest lattice spacing used in the continuum limit extrapolation $(a \sim 0.1 \mathrm{fm}$ for which $\left.m_{c} a \lesssim 1\right)$. In order to safely control this extrapolation it is thus important to asses the size of lattice artefacts affecting the observables of interest.

In the present contribution we extend the study of [1] by including a finer lattice spacing $a \simeq 0.051 \mathrm{fm}$ and we present preliminary results for the masses of some strange baryons $\left(\Omega_{s s s}, \Xi_{d s s}, \Lambda_{u d s}\right)$ and the corresponding charmed baryon obtained by substituting all the strange quarks with charm quarks $\left(\Omega_{c c c}, \Xi_{d c c}, \Lambda_{u d c}\right)$.

October 7, 2010 


\section{Setup}

As for the choice of the lattice discretization we choose here twisted mass QCD [2] with two degenerate light flavors. This regularization is attractive for its simplicity and conceptual clarity and its main advantage with respect to the standard Wilson regularization is that the spectrum and the matrix elements extracted from correlation functions are automatically $O(a)$ improved [3], i.e. lattice artefacts start at $O\left(a^{2}\right)$. The drawback is that parity and isospin are explicitly broken by $O\left(a^{2}\right)$ lattice artefacts and are recovered only in the continuum limit. The strange and charm quarks are added here only as valence quarks. We use ETMC configurations generated at three values of the lattice spacing $a \in\{0.051,0.064,0.080\} \mathrm{fm}$ and physical volumes $L \sim 2.0 \div 2.4$ fm [4]. Light sea quark masses correspond to pion masses $M_{\pi} \in[290,520] \mathrm{MeV}$ while partially quenched valence strange/charm (in the following called "heavy") quarks correspond to heavylight messon masses $M_{K} \in[520,710] \mathrm{MeV}$ and $M_{D} \in$ $[1.80,2.40] \mathrm{GeV}$. In all we have 40 different combinations $\left(M_{\pi}, M_{h l}\right)$. In order to combine data at different lattice spacings we express the value of the masses in units of $r_{0}$ [5] by multiplying the value in lattice units by the ratio $r_{0} / a$. For the three lattice spacings considered here the values $r_{0} / a \in\{8.36,6.73,5.36\}$ are taken from [4]

\section{Numerical results}

An important issue in our study is the dependence of the baryon masses upon the "heavy" quark mass $m_{h}$ in the strange and in the charm region. At $a=0.080 \mathrm{fm}$ and $M_{\pi} \simeq 340 \mathrm{MeV}$ this dependence is shown in Fig. 1 .

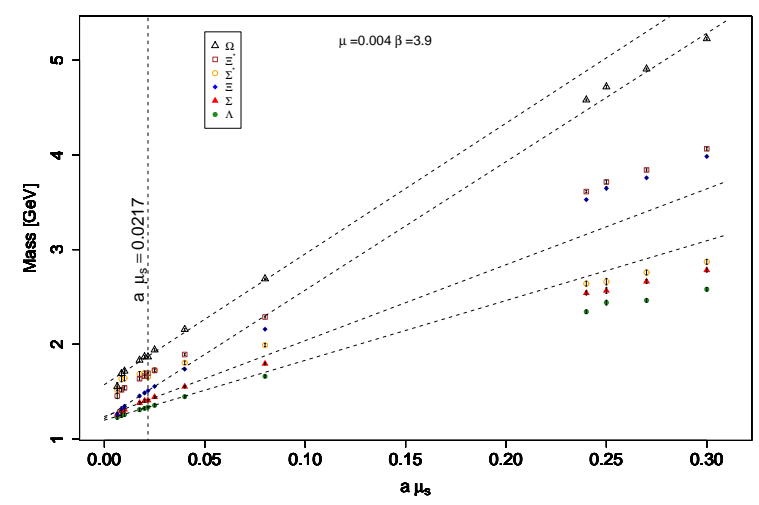

Figure 1: dependence of the octet and decuplet baryon masses on $m_{h}$. Dashed lines corespond to linear fits performed in the strange region.

As it will be confirmed in the following, Fig. 1 already suggests that baryon masses depend linearly on $m_{h}$ both in the strange and in the charm region but with two different slopes. For what concern meson masses, in the case of the Kaon we observe a dependence $M_{K}^{2} \propto m_{h}$, in agreement with the fact that the Kaon can still be considered a pseudo Goldstone boson. For the $D$ meson instead we observe a dependence $M_{D} \propto m_{h}$ as predicted by heavy quark effective theory (HQET), with no evidence of $1 / m_{h}$ term. In the following we will consider the functional dependence of baryon masses upon $M_{\pi}$ and $M_{h l}$ because this allows to extrapolate to the physical point without knowing the values of the renormalized quark masses. The observations above imply that baryon masses depend quadratically on $M_{K}$ in the strange region while depend linearly on $M_{D}$ in the charm region.

From Fig. 1 and Fig. 2 it is also evident that the splitting between $J=1 / 2$ and $J=3 / 2$ states $\left(\Sigma / \Sigma^{*}\right.$ and $\left.\Xi / \Xi^{*}\right)$ clearly diminishes with the increase of $m_{h}$. In quark models, this observation is explained thanks to the fact that the spin-spin coupling part of the $q-q$ potential is inversely proportional to the masses of the two quarks $\frac{s_{i} \mathbf{s}_{j}}{m_{i} m_{j}}$. In HQET the $\Sigma / \Sigma^{*}$ splitting is proportional to $1 / m_{h}$.

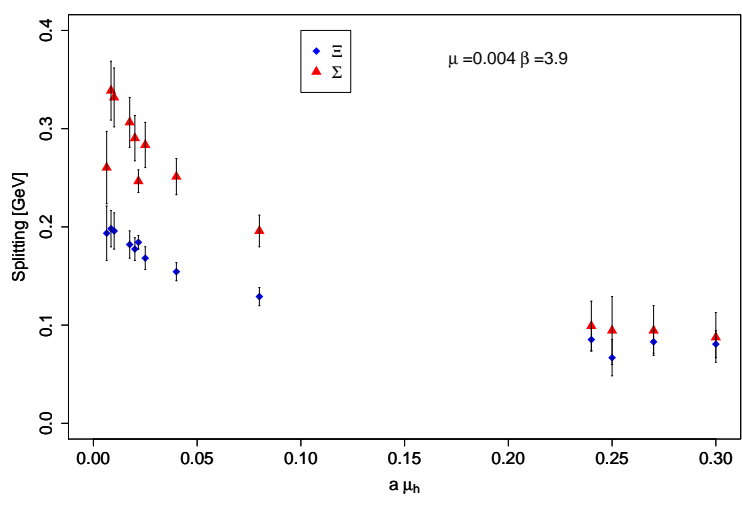

Figure 2: $\Sigma / \Sigma^{*}$ and $\Xi / \Xi^{*}$ splittings as function of $m_{h}$.

Hadron masses $M_{H}$ are extracted from the two point correlation functions $C_{\mathrm{H}}(t)=\sum_{\mathbf{x}}\left\langle H(t, \mathbf{x}) H^{\dagger}(0, \mathbf{0})\right\rangle$ of the corresponding interpolating operators $H$ at large time distances. In this region, the correlation functions decay exponentially at a rate proportional to the mass of the hadron. The value of the mass can be extracted for example by defining the effective mass $M_{\mathrm{H}}^{\text {eff }}(t)=$ $\frac{1}{a} \ln \frac{C_{\mathrm{H}}(t)}{C_{\mathrm{H}}(t+a)}$ and fitting it to a constant in the region of large time distances where the excited states have already decayed.

It turns out that the statistical error on $M_{\mathrm{H}}^{\text {eff }}(t)$ for the strange baryons grows faster in time than in the case of the charmed baryons. In the case of the $\Omega_{s s s}$ and $\Omega_{c c c}$ this is illustrated in Fig. 3. 

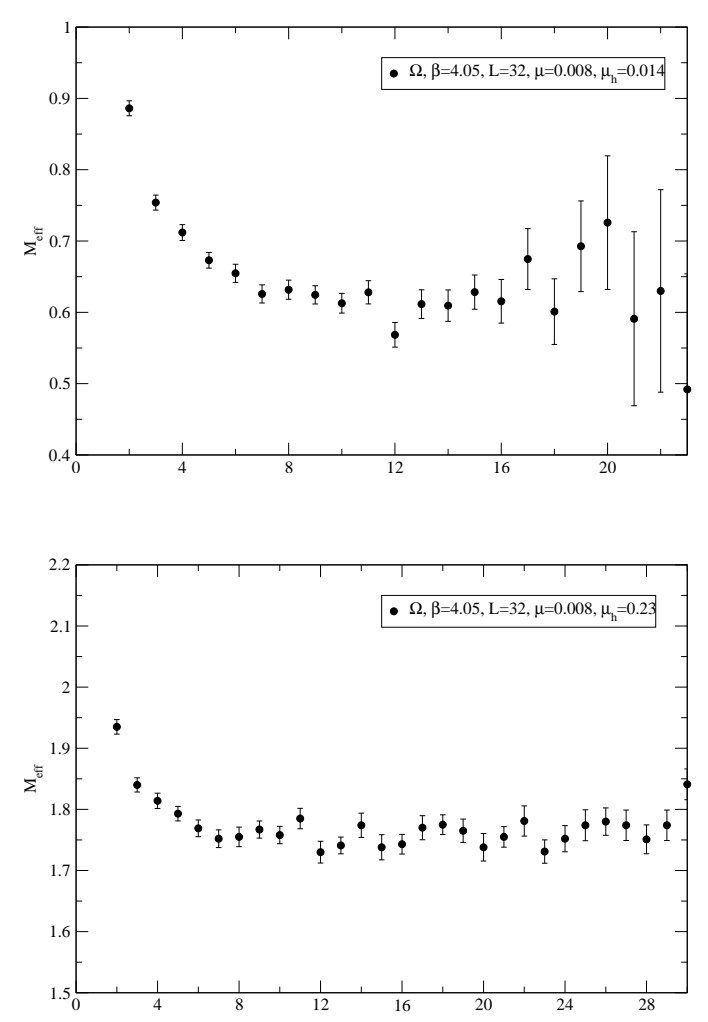

Figure 3: $M_{\Omega_{s s s}}^{\text {eff }}(t)$ (above) and $M_{\Omega_{c c c}}^{\text {eff }}(t)$ (below)

It is easy to prove that the statistical error on $M_{\Omega_{h h h}}^{\text {eff }}(t)$ is

$$
\Delta M_{\Omega_{h h h}}^{\mathrm{eff}}(t) \propto \exp \left(M_{\Omega_{h h h}}-\frac{3}{2} M_{\bar{h} h}\right) t
$$

where $M_{\bar{h} h}$ is the mass of the $\bar{h} h$ meson made of an heavy and an anti-heavy quark. This phenomenon is thus explained thanks to the observation that the ratio $M_{\bar{c} c} / M_{\Omega_{c c c}}$ has a larger value than $M_{\bar{s} s} / M_{\Omega_{s s s}}$.

\section{1. $\Omega_{\text {sss }}$ and $\Omega_{c c c}$}

In Fig. 4 we present all the 40 data points for the $\Omega$ mass fitted with a form $M_{\Omega}=\bar{M}_{0}+\bar{A} M_{\pi}^{2}+\bar{B} M_{\mathrm{hl}}^{2}+\bar{C} M_{\mathrm{hl}}^{4}$. The $\chi_{\text {d.o.f. }}^{2}$ is 1.87 and there is almost no evidence of lattice artefacts. This functional form is used only because it reduces to the form $M_{\Omega}=M_{0}+A M_{\pi}^{2}+B M_{h l}^{2}$ in the strange region while in the charm region it approximates fairly well the form $M_{\Omega}=D+E M_{\pi}^{2}+F M_{h l}$. The two forms fit well the data in the strange region (13 data points, $\left.\chi_{\text {d.o.f. }}^{2}=1.56\right)$ and charm region ( 27 data points, $\chi_{\text {d.o.f. }}^{2}=1.15$ ) respectively.

In the strange region lattice artefacts are visible and the inclusion of a term $A_{0} a^{2}$ to the functional form above lower the $\chi_{\text {d.o.f }}^{2}$ from 1.56 to 0.92 . No sign of lattice artefacts is visible instead in the charm region. This is however due to the choice of studying the behaviour of baryon masses as function of meson masses. Had we chosen to study their dependence upon the quark masses $m_{l}$ and $m_{h}$ we would have immediately remarked the presence of lattice artefacts (larger in the charm region). This is evident from the plot in Fig. 5 and in fact a fit of the form $M_{\Omega}=D+E m_{l}+F m_{h}$ which does not include lattice artefacts has a huge $\chi_{\text {d.o.f. }}^{2}=141$ while by including a term $D_{0} a^{2}$ one obtains a resonably good fit with $\chi_{\text {d.o.f. }}^{2}=1.07$. The same is valid in the strange region where the $\chi_{\text {d.o.f. }}^{2}$ reduces from 3.86 to 1.10 .

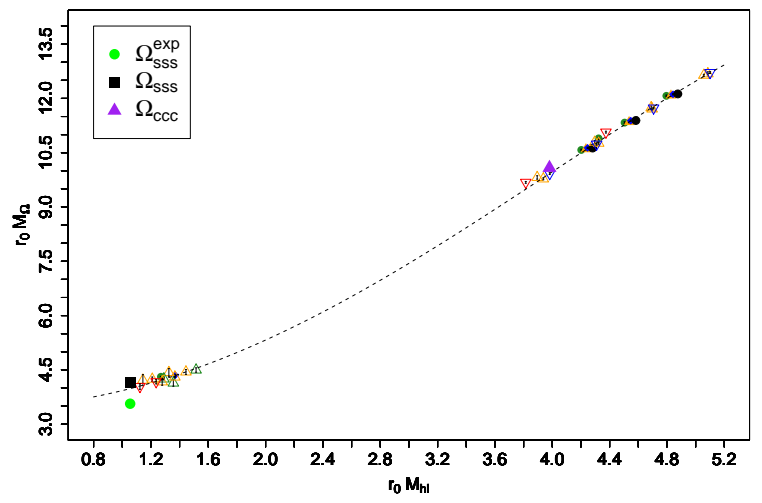

Figure 4: $M_{\Omega}$ as function of $M_{h l}$. The dashed line is a fit of the form $M_{\Omega}=$ $\bar{M}_{0}+\bar{A} M_{\pi}^{2}+\bar{B} M_{h l}^{2}+\bar{C} M_{h l}^{4}$

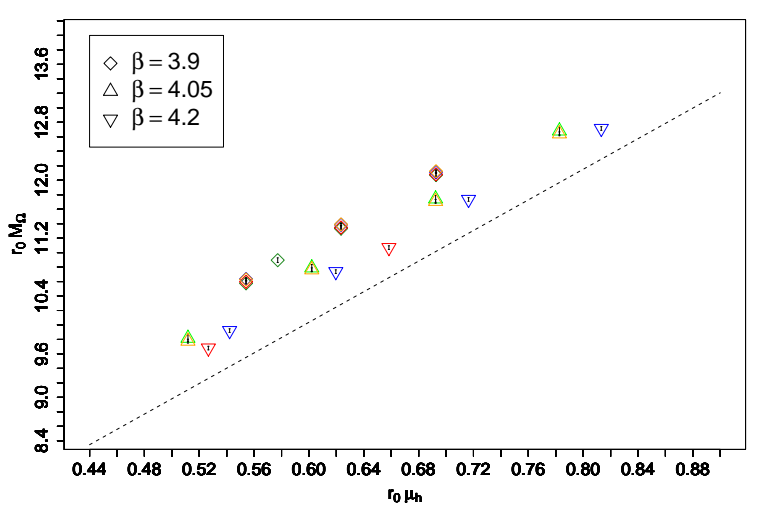

Figure 5: $M_{\Omega}$ as function of $m_{h}$ in the charm region. The dashed line is the continuum limit according to the fit form $M_{\Omega}=D+E m_{l}+F m_{h}+D_{0} a^{2}$ after setting $a=0$ and $m_{l}$ to some reference value.

We remark that $M_{\Omega}$ depends very mildly on $M_{\pi}$ and therefor the extrapolation to the physical $M_{\pi}$ seems not to pose any problem. By interpolating also to the physical value of $M_{K}$ we get the result $M_{\Omega}=1.86(20)$ $\mathrm{GeV}$ which is $10 \%$ larger than the experimental value. Due to the previous considerations and the analysis performed, this discrepancy seems not to be related to the continuum limit extrapolation or to the extrapolation in the light quark mass. Extrapolation to the physical 
$\left(M_{\pi}, M_{D}\right)$ point gives instead $M_{\Omega_{c c c}}=4.73(40) \mathrm{GeV}$ for which an experimental value is still missing.

\section{2. $\Lambda_{u d s}$ and $\Lambda_{u d c}$}

In the case of the $\Lambda$ baryon, the dependence on $M_{\pi}$ is much stronger than in the previous case and the inclusion of the term proportional to $M_{\pi}^{3}$ is crucial and reduces the $\chi_{\text {d.o.f. }}^{2}$ of a factor $\sim 0.5$ in both the strange and the charm region. Lattice artefacts are hardly visible and the functional forms we have used to fit are $M_{\Lambda}=M_{0}+A M_{\pi}^{2}+B M_{h l}^{2}+C M_{\pi}^{3}$ (in the strange region) and $M_{\Lambda}=D+E M_{\pi}^{2}+F M_{h l}+G M_{\pi}^{3}$ (in the charm region). Of course the inclusion of chiral logarithms would affect the extrapolation to the physical point. For these preliminary results we have however neglected them and performed only a rough fit using the forms written above. The dependence on $M_{\pi}$ is shown (in the charm region) in Fig. 6

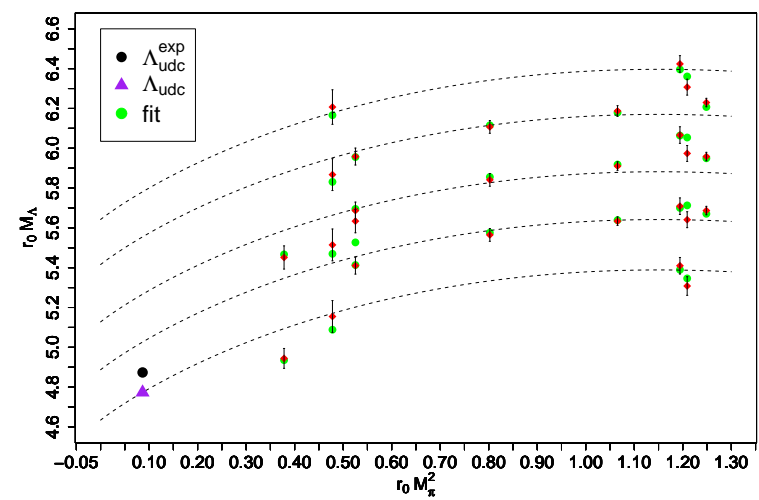

Figure 6: $M_{\Lambda_{u d c}}$ as function of $M_{\pi}^{2}$. The curves correspond to fits of the form $M_{\Lambda_{u d c}}=D+E M_{\pi}^{2}+F M_{h l}+G M_{\pi}^{3}$. They are obtained by fixing $M_{h l}$ to some values which are in general different to those of the data points.

By extrapolating to the physical $\left(M_{\pi}, M_{K}\right)$ point we obtain $M_{\Lambda_{u d s}}=1.20(10) \mathrm{GeV}$ which has to be compared with the experimental value $M_{\Lambda_{u d s}}^{\exp }=1.116 \mathrm{GeV}$. By extrapolating to the physical $\left(M_{\pi}, M_{D}\right)$ point we have $M_{\Lambda_{\text {udc }}}=2.24(18) \mathrm{GeV}$ which is in good agreement with the experimental value $M_{\Lambda_{u d c}}^{\exp }=2.286 \mathrm{GeV}$.

\section{3. $\Xi_{d s s}$ and $\Xi_{d c c}$}

Twisted mass QCD breaks explicitly isospin symmetry and thus $\Xi_{u s s}^{0}$ and $\Xi_{d s s}^{-}$(or equivalently $\Xi_{u c c}^{++}$and $\Xi_{d c c}^{+}$) are not degenerate. We thus preform a combined fit of both $\Xi_{u s s}^{0}$ and $\Xi_{d s s}^{-}$data with the form $M_{\Xi^{\{0,-\}}}=$ $M_{0}+A M_{\pi}^{2}+B M_{h l}^{2}+C M_{\pi}^{3}+A_{\{0,-\}} a^{2}$ where the coefficients $A_{\{0,-\}}$ are different for the two sets of data. Analogously we perform a combined fit of both $\Xi_{u c c}^{++}$and $\Xi_{d c c}^{+}$ data with the form $M_{\Xi^{(++,+\}}}=D+E M_{\pi}^{2}+F M_{h l}+G M_{\pi}^{3}+$ $D_{\{++,+\}} a^{2}+F_{\{++,+\}} a^{2} M_{h l}$. As in the $\Lambda$ case a term in $M_{\pi}^{3}$ is needed even though the dependence on $M_{\pi}$ is here less pronounced. Fits work well and in the continuum limit, at the physical point we get $M_{\Xi_{d s s}}=1.37(12) \mathrm{GeV}$ (to be compared with $M_{\Xi_{d s s}}^{\text {exp }}=1.32 \mathrm{GeV}$ ) and $M_{\Xi_{d c c}}=3.52(25)$ $\mathrm{GeV}$ (in perfect agreement with $M_{\Xi_{d c c}}^{\exp }=3.52 \mathrm{GeV}$ ).

\section{Conclusions}

In this preliminar study we have shown that lattice artefacts are always small in both the strange and the charm region when baryon masses are analyzed as function of meson masses. They are instead clearly visible when baryon masses are analyzed as function of quark masses. The chiral extrapolation in the light quarks confirms to be critical and a term of order $M_{\pi}^{3}$ is needed for both $\Xi$ and $\Lambda$ (it is particularly evident in this last case). $M_{\Omega_{s s s}}$ is still $10 \%$ larger than the experimental value and the source of this discrepancy seems not to be related to the continuum limit extrapolation or to the extrapolation in the light quark mass. Further investigations are needed to clarify this issue. Results for $M_{\Xi_{d s s}}, M_{\Lambda_{u d s}}, M_{\Xi_{d c c}}$ and $M_{\Lambda_{u d c}}$ nicely agree with the experimental values. We have moreover obtained a prediction for $M_{\Omega_{c c c}}=4.73(40) \mathrm{GeV}$. We are extending this preliminary analysis to the other strange and charmed baryons and also computing correlation functions to extract strange-charmed baryons. A complete analysis will be performed in the next future.

\section{Acknowledgements}

The computer time for this project was made available to us by CNRS on the BlueGene system at GENCIIDRIS (Grant 2010-052271) and CCIN2P3 in Lyon. We thank these computer centers and their staff for all technical advice and help. M. Papinutto acknowledges financial support by a Marie Curie European Reintegration Grant of the 7th European Community Framework Programme under contract number PERG05-GA-2009249309.

\section{References}

[1] Alexandrou et al. [ETMC], Phys. Rev. D 80 (2009) 114503.

[2] Frezzotti, Grassi, Sint \& Weisz, JHEP 0108 (2001) 058.

[3] Frezzotti \& Rossi, JHEP 0408 (2004) 007.

[4] Baron et al. [ETMC], JHEP 1008 (2010) 097

[5] Sommer, Nucl. Phys. B 411 (1994) 839 